\title{
Analyzing the Role of DICER1 Germline Variations in Papillary Thyroid Carcinoma
}

\author{
Sule Canberk ${ }^{a, b, d}$ Joana C. Ferreira ${ }^{a, b}$ Luísa Pereira ${ }^{a, b, c}$ Rui Batısta ${ }^{a, b}$ \\ Andre F. Vieira ${ }^{a, b}$ Paula Soares ${ }^{a, b, c}$ Manuel Sobrinho Simões ${ }^{a, b}, c, d, e$ \\ Valdemar Máximo a, b, c

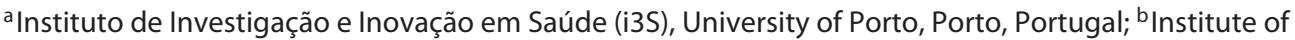 \\ Molecular Pathology and Immunology of the University of Porto (Ipatimup), Porto, Portugal; \\ 'Department of Pathology, Faculty of Medicine of the University of Porto (FMUP), Porto, Portugal; \\ ${ }^{\mathrm{d}}$ Abel Salazar Biomedical Sciences Institute (ICBAS), University of Porto, Porto, Portugal; ${ }^{e}$ Department of \\ Pathology and Oncology, Centro Hospitalar São João, Porto, Portugal
}

\section{Keywords}

DICER1 - Papillary thyroid carcinoma - Germline variants · Somatic mutation - Thyroid - Paediatric thyroid nodules . Syndromic thyroid disease $\cdot$ Familial thyroid disease

\footnotetext{
Abstract

Introduction: DICER1 is a member of RNase III family that has a pivotal role in the biogenesis of microRNAs, being important for normal development. Dysregulation of DICER1 has been described in different human tumours; however, there is insufficient data on the risk of thyroid cancer in the presence of germline DICER1 variants, particularly when focusing on the background of papillary thyroid carcinoma (PTC). For this purpose, we ascertained the presence of DICER1 variants in 502 (PTC) cases available from The Cancer Genome Atlas (TCGA) research network in a well-characterized pathological context. Material and Methods: in this study we analyzed 502 samples from 502 patients, described as PTC in the TCGA database. Tumour diagnoses were re-evaluated by 2 pathologists (S.C. and M.S.-S.) on slides available
}

from the database, and clinicopathological and demographic data was examined. Data concerning germline and sporadic DICER1 gene variants as well as frequent mutations in the genes involved in thyroid carcinogenesis (e.g., RAS and BRAF V600E) was retrieved from the database. Results and Discussion: We report 1 new germline possibly pathogenic variant, besides 15 others already been identified in ClinVar. We found that the DICER1-positive PTC group more frequently includes PTC variants, namely the oncocytic, follicular, and aggressive (hobnail variant of PTC) variants. A previous association of DICER1 had been demonstrated, mainly with the follicular variant of PTC and follicular thyroid carcinomas. Tumours harbouring germline DICER1 mutations were more frequently "bilateral" and "encapsulated." The frequent association of DICER1 germline variants with other mutations associated with thyroid cancer can reflect an haploinsufficiency tumour suppressor gene function of DICER1, as suggested from the study of animal models.

(C) 2020 European Thyroid Association Published by S. Karger AG, Basel

\footnotetext{
karger@karger.com

www.karger.com/et

(c) 2020 European Thyroid Association

Published by S. Karger AG, Basel
}

Karger
Valdemar Máximo

Instituto de Investigação e Inovação em Saúde (i3S), University of Porto Rua Alfredo Allen 208 PT-4200-135 Porto (Portugal)

vmaximo@ipatimup.pt 


\section{Introduction}

Thyroid cancer is the most common of all endocrine malignancies. Despite the advances in its genetic characterization, many authors now accept that about $30 \%$ of thyroid cancer cases are in a genetic "dark zone" due to the lack of well-established driver mutations [1,2]. Papillary thyroid carcinoma (PTC), the most common type (i.e., $90 \%$ of all thyroid malignancies) probably contributes to most such tumours in this "dark zone" [3].

Approximately $90 \%$ of thyroid cancers occur sporadically, but $3-9 \%$ are defined as familial non-medullary thyroid cancer (FNMTC). Less than 5\% of FNMTC is seen in syndromic forms associated with well-defined driver germline alterations. This rare type of FNMTC includes Cowden syndrome, familial adenomatous polyposis, Gardner syndrome, Carney complex type 1, Werner syndrome, and DICER1 syndrome. The remaining 95\% of FNMTCs represent a less-defined genetic susceptibility group, designated as "non-syndromic." Of these, DICER1 (previously known as "MNG1 [14q32]) represents a group of rare diseases, seen in patients that are either "syndromic," with a predominance of non-thyroidal tumours, or "non-syndromic" in which thyroid cancer is the main component $[4,5]$. Some "syndromic" patients present with thyroid disease before the aggressive high-grade malignant tumour occurs in other organs (non-thyroidal tumours). This phase may be called the "pre-syndromic/indecisive" period (online suppl. Fig. 1; see www.karger.com/doi/10.1159/000509183 for all online suppl. material).

DICER1 is a member of the RNAse III family that plays a key role in the biogenesis involved in the processing of small non-coding RNAs (microRNAs), potentially affecting gene regulation at the post-transcriptional level. Since the first report in 2001 [6], increasing attention has been paid to DICER1 involvement in cancer, particularly hereditary syndromic forms. The verification that DICER1 germline variations result in a distinctive phenotype was reported by Hill et al. [7] in a case involving pleuropulmonary blastoma (PPB); subsequently, Slade et al. [8] detected DICER1 germline mutations in 19/823 unrelated patients with a broad range of tumours (http://www. ppbregistry.org/pdf/Doc_D.pdf). Based on the presence of a variety of childhood tumours (pleuropulmonary blastoma, cystic nephroma, Sertoli-Leydig cell tumours, embryonal rhabdomyosarcoma, etc.), the authors proposed the term "DICER1 pleiotropic tumour predisposition syndrome" for this entity $[7,8]$. However, none of the pioneer studies highlighted the presence of thyroid lesions in this setting. Later, Rio Frio et al. [9] identified 5 different heterozygous mutations in the DICER1 gene in 5 families showing autosomal dominant MNG, with or without Sertoli-Leydig cell tumours. Their results extended the tumour spectrum beyond the lung, kidney, and other well-described childhood tumours, and encouraged interest in thyroid lesions.

DICER1 mutations (germline and/or somatic) have been described in thyroid tumours, mostly in multinodular goitre (MNG), follicular adenoma (FA), and follicular thyroid carcinoma (FTC) [2]. However, the role of DICER1 variants in PTC and the clinicopathological implications are largely unexplored.

To assess the contribution of DICER1 variants in thyroid cancer predisposition, particularly in PTC, we analyzed the prevalence of germline/somatic variants in the largest PTC case series available in The Cancer Genome Atlas (TCGA), the most comprehensive multiplatform data portal.

\section{Materials and Methods}

TCGA is a collaboration between the National Cancer Institute (NCI) and the National Human Genome Research Institute (NHGRI). It assembles genomic and transcriptomic data of the largest tumour collection (https:// cancergenome.nih.gov/) of PTCs. Histological slides and clinicopathological information were retrieved from the medical records of each of the 502 patients, and genetic information (about thyroid cancer) was obtained from the TCGA data portal (access licence: project No. 6494).

\section{Germline Variant Classification}

The TCGA data was filtered for the detection of DICER1 germline variants by a 2 -step filtering process.

First, exome-aligned sequence data from non-tumour samples (mostly from the blood, and, when not available, from thyroid samples) in the BAM file format, aligned to the GRCh37 human reference genome, and the accompanying meta-data were downloaded from the genomic data common website. Reads spanning the DICER1 gene were obtained by means of SAMtools [10] and variant calling with the GATK HaplotypeCaller v3.4 tool, following current best practice [11]. Only variants with a mean depth $>15$ were considered in this analysis and used in the second filtering step. A total of 215 variants, all with an rs number, were found in the DICER1 gene; 123 appeared in the 1st filtering step (L.P. and J.C.F.), and the other 92 required further analysis in the 2 nd filtering step (R.B.). Seven germline variants (7/123) were identified in the 1 st step and 9 (9/92) in the 2nd step. In total, from TCGA, we found 16 germline variants in 18 cases that could potentially have a functional impact. Based on this premise, synonymous and intronic variants were excluded, and the remaining alterations (14 missense and 2 small deletions) with an overall minimum allele frequency (MAF) $<0.02$ (compiled from the former ExAC database excluding TCGA data, and now aggregated on the gnomAD database; https:/gnomad.broadinstitute.org/) were selected for 
further study. The selected variants were also documented by at least 2 predictive deleteriousness scoring methods (PolyPhen-2, SIFT, MutationTaster) and the NIH-based database, ClinVar (Table $1 \mathrm{~A})$.

As the result of the 2 filtering steps, 16 variants were found to match the criteria described above; these are referred to as "possibly pathogenic" in our analysis.

Somatic mutations of the DICER 1 gene were downloaded from TCGA website and correspond with those reported in a previous paper by TCGA Consortium on thyroid carcinoma (Table 1B) [1].

Since PTC is a MAPK-driven cancer, the BRAF V600E and RAS gene (N-RAS, H-RAS, and K-RAS) mutations were also filtered from TCGA data for the entire dataset, in addition to other mutations with a lower prevalence which are clustered in the group "other alterations" (e.g., rare somatic mutations, gene rearrangements, or fusion genes).

\section{Group Definition}

For further analysis, only filtered DICER1 germline variants were considered. The 2 main groups were defined "DICER1 positive" ( $n=18)$ and "DICER1 negative" $(n=472)$. The DICER1 positive group comprised cases that presented germline variants that passed our previous filtering (explained above in detail). The remaining cases were included in the "DICER1 negative group."

Thirty-eight papers were included in our review of the literature (online suppl. Table 1). The cases reporting family history, DICER1 germline variants, and at least one associated tumour previously reported in DICER1 syndrome were classified as "syndromic." Carriers or non-carriers with thyroid disease only (with no evidence of multiple tumours) were classified as "non-syndromic."

Evaluation of Clinicopathological Baseline Characteristics

The digital slides from 502 tissue samples available from TCGA database were re-evaluated by 2 pathologists (S.C. and M.S.-S.) and reclassified based on the WHO criteria when they were considered representative [12]. Twelve cases were excluded from the study due to a lack of representative material and for not matching the PTC diagnostic criteria.

Clinicopathological variables were retrieved from TCGA database when available. The detailed pathological characteristics were searched and recorded from 490 patients' pathology reports as provided by TCGA registry. Fifteen variables (13 clinicopathological and 2 demographic) were included in the study: age, gender, lobe, diagnosis/variant classification, tumour size, extrathyroidal extension (ETE) minimal and gross, angiolymphatic invasion (ALI), capsule invasion (CI), laterality (L), lymph node metastasis (LNM), focality (F), distant metastasis (DM), and residual tumour. The term "non-variant" is reserved for classic PTC only, and any variants other than classic PTC were grouped under the term "variant."

\section{Statistical Analysis}

Statistical analysis was done using the SPSS statistical software v20.0 (Chicago, IL, USA). Qualitative variables are shown as percentages. To assess significant differences in the DICER1 positive and DICER1 negative groups with regard to pathological, clinical, and genetic variables, we used two-way contingency tables and the $\chi^{2}$ or Fisher's exact test when appropriate. We considered $p<0.05$ to be statistically significant.

\section{Results}

After histological revision, the 490 thyroid cases evaluated were: 162 (33\%) classic variant (CV-PTC), 156 (31\%) oncocytic variant (OV-PTC), 131 (26\%) follicular variant (FV-PTC), 13 (3\%) hobnail variant (HV-PTC), 12 (2\%) Warthin-like (WL-PTC), 7 (1.4\%) tall-cell variant (TCVPTC), 5 (1\%) columnar-cell variant (CCV-PTC), and 4 $(0.8 \%)$ other very rare variants of PTC. In total, 157 germline variants in DICER1 were filtered from TCGA data, and after the 2 steps of filtering (Material and Methods), 16 germline variants in 18 cases remained for subsequent study (Table 1A). Fourteen germline variants were missense (leading to an amino acid change) and 2 were small deletions. Of the 16 variants, 7 were described as benign, 2 as likely benign, 3 as possibly damaging, and 4 had no classification by predictive scoring methods (PolyPhen-2, SIFT, and MutationTaster; Table 1A). Fifteen variants had already been described in the variant classifier NIHbased database ClinVar (Table 1A), 6 as benign/likely, 1 as benign, and the remaining 8 as being of uncertain significance. Besides these 15, we reported 1 new germline alteration, c. $2512 \mathrm{~T}>\mathrm{G}$ (p. Leu838Val), that is possibly associated with disease (Table 1A).

One variant was recurrently found in 3 cases. We evaluated the probability of the biological impact of these variants by predictive scoring methods (PolyPhen-2, SIFT, and MutationTaster; Table 1A). Five cases, 3 with the same recurrent variant, c.2951A $>C$ (p. Asn984Thr), presented variants that were classified as possibly damaging. The relevant clinicopathological, genetic, and demographic data on cases showing this recurrent variant are summarized in Table 2. This recurrent variant, c.2951A $>C$ (p. Asn984Thr), had been submitted to ClinVar with 3 conditions; 2 were reported as hereditary nonpolyposis colon cancer (Lynch syndrome). The other 2 variants classified as possibly damaging were detected in a female with unilateral disease (64 years of age and harbouring a nodule $13 \mathrm{~mm}$ in size) and a male with bilateral disease (44 years of age and harbouring a nodule $21 \mathrm{~mm}$ in size) and encapsulation and capsular invasion were evident. The only bilateral case was reported with lymph node metastasis. None of the germline variants were associated with DICER1 somatic mutations in this group. Three somatic DICER1 mutations were detected in 3 other cases previously described in the literature [2]; there were no germline variants associated with these somatic DICER1 mutations (Table 1B).

The oncocytic phenotype or oncocytic features (OVPTC and HV-PTC) were observed in 9 cases (43\%; 9/21) 
III

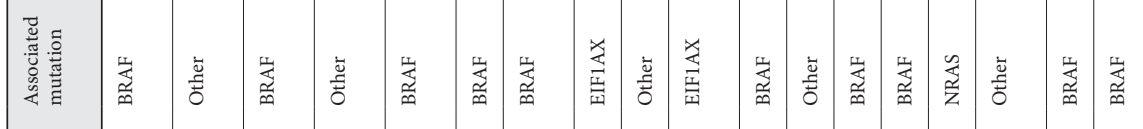
经: H

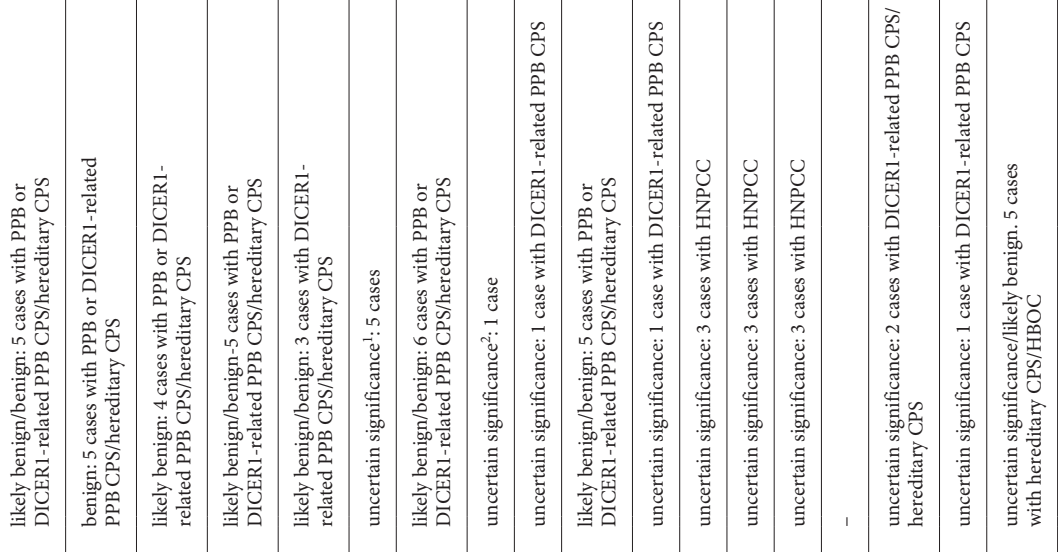

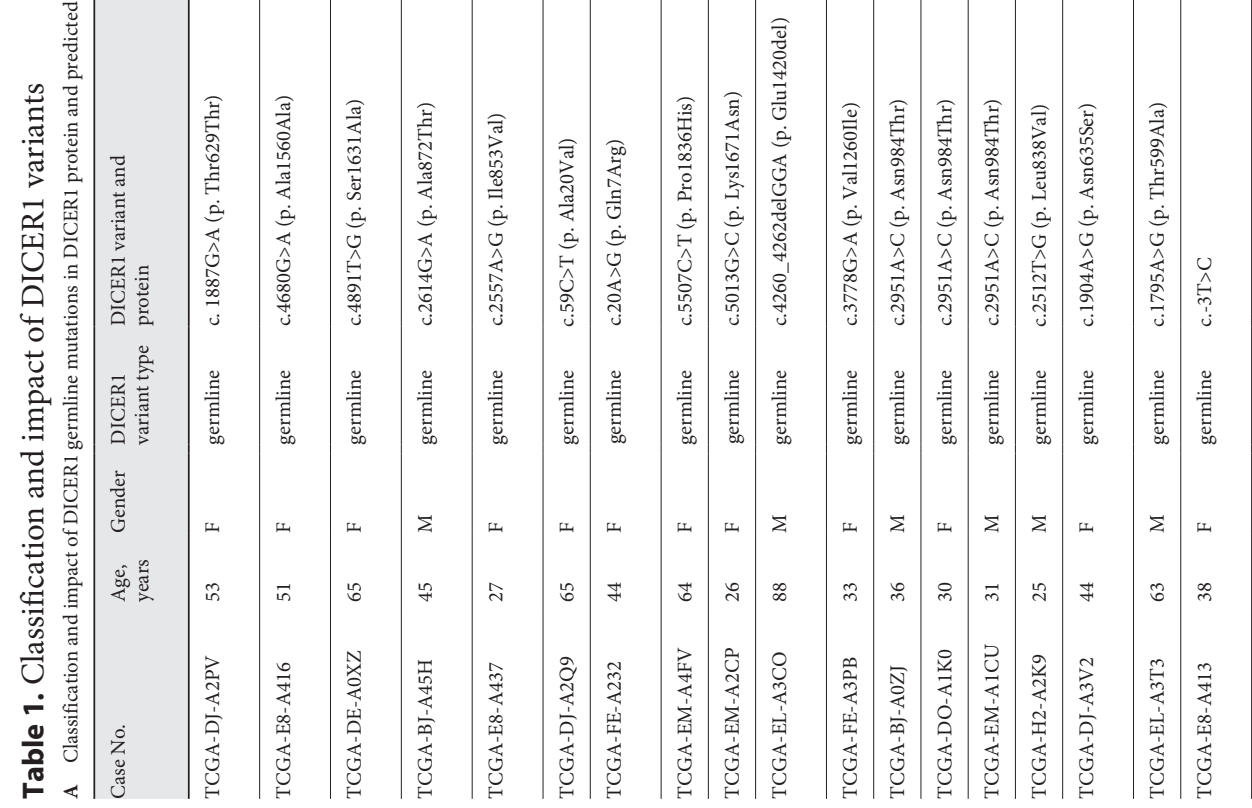

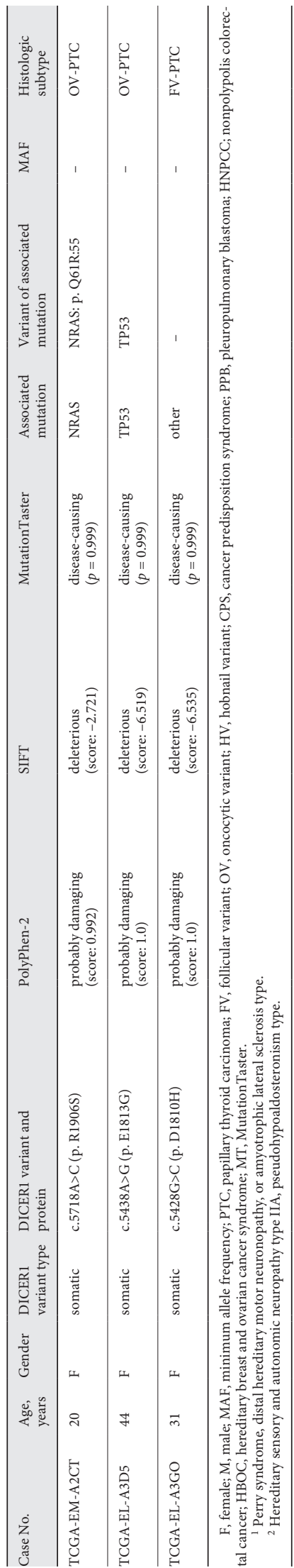


Table 2. Clinicopathological and genetic profile of the recurrent variant c.2951A $>C$ (p. Asn984Thr)

\begin{tabular}{llll}
\hline & TCGA-BJ-A0ZJ & TCGA-DO-A1K0 & TCGA-EM-A1CU \\
\hline Age, years & 36 & 30 & 31 \\
Gender & male & female & male \\
Size, mm & 45 & 42 & 65 \\
Laterality & bilateral & bilateral & bilateral \\
Focality & multifocal & multifocal & multifocal \\
Variant type & FV-PTC & classic PTC & classic PTC \\
Presence of capsule & Yes & Yes & Yes \\
Capsule invasion & Yes & Yes & Yes \\
Lymph node metastasis & $1 / 8$ & $9 / 14$ & $1 / 20$ \\
Extranodal involvement & No & Yes & No \\
Angiolymphatic invasion & Yes & n.a. & n.a. \\
Minimal extrathyroidal extension & No & Yes & No \\
Additional genetic events & other & BRAF & BRAF \\
\hline
\end{tabular}

PTC, papillary thyroid carcinoma; FV, follicular variant; n.a., not available.

Table 3. Results based on pathological, genetic, and clinical analysis in DICER1 positive and DICER1 negative groups

\begin{tabular}{lccc}
\hline Pathological characteristics & DICER1 positive & DICER1 negative & $p$ value \\
\hline Presence of capsule & 1 & 100 & \\
$\quad$ No & 13 & 174 & 0.02 \\
$\quad$ Partially/Yes & 8 & 393 & \\
Laterality & 9 & 72 & $<0.01$ \\
$\quad$ Unilateral & & & \\
$\quad$ Bilateral & & & \\
\hline
\end{tabular}

of all DICER1 groups including germline and somatic variants. Seven cases were in the group with germline alterations $(39 \% ; 7 / 18)$ and 2 were in the group with DICER1 somatic mutations (67\%; $2 / 3$; Table 1$)$.

Additional genetic events were available for 410 cases in the dataset. All the cases with either germline $(n=18)$ or somatic $(n=3)$ DICER1 alterations harboured additional mutations $(100 \% ; 21 / 21)$. Thirteen cases $(72 \%$; $13 / 18)$ in the germline group and $2(67 \% ; 2 / 3)$ in the somatic group were found in genes that commonly mutate in thyroid cancer (BRAF, RAS, EIF1AX, and TP53) (Table $1)$. This was in contrast with the DICER1 negative cases where additional mutations were found in 83\% (389/469) of the cases.

Among the 13 clinicopathological variables studied, data on laterality (bilaterality vs. unilaterality) was available for 484 cases, and bilateral tumours were significantly more frequent in the DICER1 positive group than in the DICER1 negative group (53\% [9/17] vs. 15\% [72/467], $p<0.01$ ). For 288 cases, "presence of capsule" (encapsula- tion vs. lack of capsule) data was available, and encapsulated tumours were significantly more frequent in the DICER1 positive group (92.8\% [13/14], vs. 36\% [100/274], $p<0.02$ ) (Table 3 ).

Data on angiolymphatic invasion (present or absent) was available for 383 cases, and 111 were reported as having angiolymphatic invasion. There was data on only 2 tumours in the DICER 1 positive group, and both had angiolymphatic invasion; the remaining 109 cases were from the DICER1 negative group (100\% [2/2] vs. $28.6 \%[109 / 381], p<0.08)$. Although there was limited available information for the DICER1 positive group (only 2 cases), there was a slight tendency towards significance.

The clinicopathological and genetic profiles collected for DICER1 (germline) positive group were also analyzed in the 3 cases of DICER1 somatic mutation and are summarized in Table 4 . Of these 3 cases, 2 were reported as unilateral and unifocal. 
Table 4. Clinicopathological and genetic profile of the cases with DICER1 somatic mutations

\begin{tabular}{llll}
\hline & $\begin{array}{l}\text { TCGA- } \\
\text { EM-A2CT }\end{array}$ & $\begin{array}{l}\text { TCGA- } \\
\text { EL-A3D5 }\end{array}$ & $\begin{array}{l}\text { TCGA- } \\
\text { EL-A3GO }\end{array}$ \\
\hline Age, years & 20 & 44 & 31 \\
Gender & female & female & female \\
Size, mm & 11 & 60 & 32 \\
Laterality & unilateral & n.a. & unilateral \\
Focality & unifocal & multifocal & unifocal \\
Variant type & OV-PTC & OV-PTC & FV-PTC \\
Presence of capsule & partially & n.a. & n.a. \\
Capsule invasion & No & n.a. & n.a. \\
Lymph node metastasis & $0 / 5$ & $15 / 30$ & $0 / 4$ \\
Extranodal involvement & No & Yes & No \\
Angiolymphatic invasion & n.a. & No & n.a. \\
Minimal ETE & No & Yes & No \\
Additional genetic events & NRAS & TP53 & other \\
& & & \\
\hline
\end{tabular}

PTC, papillary thyroid carcinoma; FV, follicular variant; OV oncocytic variant; ETE, extrathyroidal extension; n.a., not available.

\section{Discussion}

Our review of the literature showed that, despite the variety of clinical presentations in the DICER1 "syndromic" and "non-syndromic" groups, the most common disease is thyroid neoplasm, which led some authors to endorse the thyroid manifestation as a clinical marker of these 2 groups (online suppl. Table 1) [13, 14].

In a previous study, Kim et al. [15] analyzed the prevalence of DICER1 pathogenic variations in population databases, excluding TCGA cases, and showed that $95 \%$ of the children with a DICER1 pathogenic variant do not develop any malignancy or tumour by the age of 10 years. This data suggests that germline pathogenic DICER1 variants may be more common than the rare manifestation in a syndromic fashion. In our review, we identified a total of 172 thyroid tumours associated with DICER1; $40 \%$ of the cases were syndromic $(70 / 172)$ and $60 \%$ were non-syndromic (102/172) (online suppl. Table 1). In these 172 cases, the risk of malignancy was found to be $67 \%(47 / 70)$ and $75 \%(77 / 102)$ in the syndromic and nonsyndromic groups, respectively.

In Kim et al. [15], 147 unique missense variants were found. Except for the p. Lys1671Asn, p. Val1260Ile, and p. Leu838Val mutations, all 15 variants that we found were also reported in their study.

In our study, to investigate the role of DICER 1 mutations/variants in PTC, we had the advantage of having access to data from a large series of PTC (TCGA database encompassing 502 PTC) that included digital histological slides and clinicopathological data. We confirmed the presence of the 3 DICER1 somatic mutations (2 OV-PTC and 1 FV-PTC) previously reported in TCGA database (Table 1B) [2]. One is associated with N-RAS, one with TP53, and the remaining case with an additional and rare somatic mutation. Subsequent analysis showed no additional germline DICER1 mutation in these 3 cases. Two were unilateral and unifocal, and there was no presence of capsule data in 2 of them (Table 4).

Using the 2 filtering steps described in Material and Methods, 16 DICER1 germline variants were found in 18 cases of PTC in our study. These variants present a frequency, in human DNA databases, that rules out the definition of common polymorphism. For these reasons, we decided to call them "possibly pathogenic" and selected them for further analysis.

Histologically, DICER1-related thyroid lesions are known to be either benign or low-risk malignancies (mainly MNG, FA, and minimally invasive FTC) [2]. In more recent studies, a greater frequency of FV-PTC has been reported (online suppl. Table 1). In our series, we noted a high prevalence of tumours with oncocytic features in the DICER1 positive cases that was not documented previously (Table 1). Poiana et al. [16] reported a cystic PTC with oxyphilia with SLCT, and Pinto et al. [17] a FA with oncocytic morphology. The alterations published in the latest WHO publication could be the basis of this discrepancy, since these OV-PTCs were placed in the category FV-PTC without including the presence of oncocytic morphology [18]. If we merge OV-PTC cases (6/18) with FV-PTC cases (5/18), the most common PTC variant in the DICER1 positive group would be FV-PTC $(11 / 18)$.

Interestingly, and similarly to what was observed in the cases with somatic mutation, all the DICER1 germline variants found were concomitant with additional thyroid cancer canonical mutations, and with no loss of the second allele (Table 1). Thus, as demonstrated previously in mouse models, the role of DICER1 in tumorigenesis does not match the classic definition of either a tumour suppressor gene or an oncogene $[19,20]$. While the loss of a single allele of DICER1 promotes tumorigenesis, the loss of both alleles has an inhibitory effect [20]. There is another hypothesis that can explain the mechanism behind the role of DICER1 (online suppl. Fig. 2). Apparently, $\geq 1$ additional genetic events (in other genes) are required to promote tumorigenesis [20]. To the best of our knowledge, we describe here for the first time the association of well-known thyroid cancer mutations (RAS/RAF path- 
way) and DICER1 germline alterations, thereby reinforcing the role of tumour haploinsufficiency of DICER1. The bi-allelic loss of DICER 1 should be explored as a therapeutic target, given the evidence that homozygotic loss leads to cell death $[19,20]$.

Our data shows that the presence of DICER1 germline alterations did not correlate with age and gender, but rather with the features tumour bilaterality and presence of capsule. Many studies have stated that despite the likely high penetrance of benign thyroid nodules in DICER1 syndrome, only a small percentage will progress to thyroid carcinoma. However, in a nicely designed study, Khan et al. [13] demonstrated a 16- to 18-fold increased risk of thyroid cancer by the age of 40 years in a very large, family-based cohort of individuals with DICER1 syndrome.

Despite the female predominance in the background of DICER1 in the literature (online suppl. Table 1), our study and that of Pinto et al. [17] did not find any association with gender.

Bilaterality is a well-known feature associated with FNMTC, and our results suggest that presence of capsule can also be an indicator of the DICER1 familial origin of these tumours (unpublish. pers. obs.), but the available information is scarce and restrains further interpretation. Regardless of the population type, i.e., either syndromic or non-syndromic, it is reported that bilaterality is one of the predictive factors for central lymph node metastasis in PTC which is a risk factor for disease recurrence [21, 22]. These results, together with previous reports, strengthen the current argument for active surveillance of patients with DICER1 manifestation $[8,13,14]$.

Our study has limitations. First is the absence of familial information in the cases with germline DICER1 variants. Second, variant filtering encompasses the inevitable risk of losing important information (pathogenic variants) that did not pass initial filters, and prediction algorithms show limitations regarding the protein function impairment of the analyzed variants. Finally, details were missing and/or not updated in the pathological and clinical characteristics of the available data.

Nonetheless, multi-faceted documentation of DICER1 is only now beginning to emerge. Our data presented here is an approach to DICER1 alterations in apparently sporadic PTC and raises some "opening" questions that might lead to future studies: (1) the phenotype of thyroid malignancy in the background of DICER1 carriers, (2) the frequency of additional genetic events in DICER1 carriers who have thyroid malignancy, (3) the histopathological profile of thyroid malignancies in syndromic, non-syn- dromic, and/or presyndromic/indecisive forms and the clinical importance of screening and treating in those groups, and (4) the prevalence of non-invasive follicular thyroid neoplasm with papillary-like nuclear features (NIFTP) or other encapsulated thyroid tumours in this context, as endorsed in 2 separate chapter by the 4 th edition of the WHO endocrine blue book [12]. As we evaluated TCGA cases based on single H\&E digital slides, we could not address the meaning of such encapsulated lesions using any of the established criteria. Although NIFTP was not considered in previous studies, most of the PTCs previously published related to DICER1 were classified as encapsulated FV-PTC or FV-PTC. Taking all this together, at least some of the aforementioned DICER1 PTCs may correspond with NIFTP or "other encapsulated thyroid tumours."

We report 1 new germline variant, c.2512T $>\mathrm{G}$ (p. Leu $838 \mathrm{Val}$ ), as possibly pathogenic besides 15 others which had already been defined in ClinVar (Table 1A). The data also shows the association of conventional genetic mutations along with DICER1 variants which underlines the tumour haploinsufficiency role of DICER1.

\section{Acknowledgements}

We wish to thank TCGA for the access provided to all the protected data required specifically for this work.

\section{Statement of Ethics}

This study was performed in accordance with national regulatory laws for the handling of biological specimens from repositories/tumour banks, the samples being exclusively available for research purposes in retrospective studies, as well as under the International Helsinki Declaration.

\section{Conflict of Interest Statement}

The authors have no conflicts of interest to declare.

\section{Funding Sources}

This work was supported by Portuguese funds through FCT Fundação para a Ciência e a Tecnologia - in the framework of project UID/BIM/04293/2013 and through a $\mathrm{PhD}$ grant to $\mathrm{RB}$ SFRH/BD/111321/2015 and SC (SFRH/BD/147650/2019). It was also financed by FEDER - Fundo Europeu de Desenvolvimento Regional funds through the COMPETE 2020 - Operational Program for Competitiveness and Internationalization (POCI), Por- 
tugal 2020, and by Portuguese funds through FCT - Fundação para a Ciência e Tecnologia/ Ministério da Ciência, Tecnologia e Inovação in the framework of the projects "Institute for Research and Innovation in Health Sciences" (POCI-01-0145-FEDER-007274). Further funding by the European Regional Development Fund (ERDF) through the Operational Programme for Competitiveness and Internationalisation - COMPETE 2020, and Portuguese national funds via FCT, under project POCI01-0145-FEDER-016390: CANCEL STEM.

\section{Author Contributions}

M.S.-S., P.S., S.C., and V.M.: conceptualization. S.C., J.C.F., R.B., and L.P.: data processing. A.F.V. and S.C.: statistics. S.C.: writing of the original draft. P.S., S.S., A.V., L.P., S.C., and V.M.: writing, review, and editing. V.M.: supervision.

\section{References}

1 Agrawal N, Akbani R, Aksoy BA, Ally A, Arachchi H, Asa SL, et al.; Cancer Genome Atlas Research Network. Integrated genomic characterization of papillary thyroid carcinoma. Cell. 2014 Oct;159(3):676-90.

2 Wasserman JD, Sabbaghian N, Fahiminiya S, Chami R, Mete O, Acker M, et al. DICER1 Mutations Are Frequent in Adolescent-Onset Papillary Thyroid Carcinoma. J Clin Endocrinol Metab. 2018 May;103(5):2009-15.

3 Costa V, Esposito R, Pallante P, Ciccodicola A, Fusco A. The "next-generation" knowledge of papillary thyroid carcinoma. Cell Cycle. 2015;14(13):2018-21.

4 Peiling Yang S, Ngeow J. Familial non-medullary thyroid cancer: unraveling the genetic maze. Endocr Relat Cancer. 2016 Dec; 23(12):R577-95.

5 Couch RM, Hughes IA, DeSa DJ, Schiffrin A, Guyda H, Winter IS. An autosomal dominant form of adolescent multinodular goiter. Am J Hum Genet. 1986 Dec;39(6):811-6.

6 Hill DA, Sadeghi S, Schultz MZ, Burr JS, Dehner LP. Pleuropulmonary blastoma in an adult: an initial case report. Cancer. 1999 Jun; 85(11):2368-74.

7 Hill DA, Ivanovich J, Priest JR, Gurnett CA, Dehner LP, Desruisseau D, et al. DICER1 mutations in familial pleuropulmonary blastoma. Science. 2009 Aug;325(5943):965.

8 Slade I, Bacchelli C, Davies H, Murray A, Abbaszadeh F, Hanks S, et al. DICER1 syndrome: clarifying the diagnosis, clinical features and management implications of a pleiotropic tumour predisposition syndrome. J Med Genet. 2011 Apr;48(4):273-8.
9 Rio Frio T, Bahubeshi A, Kanellopoulou C, Hamel N, Niedziela M, Sabbaghian N, et al. DICER1 mutations in familial multinodular goiter with and without ovarian Sertoli-Leydig cell tumors. JAMA. 2011 Jan;305(1):6877.

10 Li H, Handsaker B, Wysoker A, Fennell T, Ruan J, Homer N, et al.; 1000 Genome Project Data Processing Subgroup. The Sequence Alignment/Map format and SAMtools. Bioinformatics. 2009 Aug;25(16):2078-9.

11 DePristo MA, Banks E, Poplin R, Garimella KV, Maguire JR, Hartl C, et al. A framework for variation discovery and genotyping using next-generation DNA sequencing data. Nat Genet. 2011 May;43(5):491-8.

12 Lloyd RV, Osamura YR, Kloppel G, Rosai J, editors. WHO Classification of Tumours of Endocrine Organs. 4th ed. Lyon: IARC; 2017. pp. 78-80.

13 Khan NE, Bauer AJ, Schultz KA, Doros L, Decastro RM, Ling A, et al. Quantification of Thyroid Cancer and Multinodular Goiter Risk in the DICER1 Syndrome: A FamilyBased Cohort Study. J Clin Endocrinol Metab. 2017 May; 102(5):1614-22.

14 de Kock L, Sabbaghian N, Soglio DB, Guillerman RP, Park BK, Chami R, et al. Exploring the association Between DICER1 mutations and differentiated thyroid carcinoma. J Clin Endocrinol Metab. 2014 Jun; 99(6):E1072-7.

15 Kim J, Field A, Schultz KA, Hill DA, Stewart DR. The prevalence of DICER1 pathogenic variation in population databases. Int J Cancer. 2017 Nov;141(10):2030-6.
16 Poiana C, Virtej I, Carsote M, Banceanu G, Sajin M, Stanescu B, et al. Virilising SertoliLeydig cell tumour associated with thyroid papillary carcinoma: case report and general considerations. Gynecol Endocrinol. 2010 Aug;26(8):617-22.

17 Pinto AE, Silva GL, Henrique R, Menezes FD, Teixeira MR, Leite V, et al. Familial vs. sporadic papillary thyroid carcinoma: a matchedcase comparative study showing similar clinical/prognostic behaviour. Eur J Endocrinol. 2013 Dec;170(2):321-7.

18 Canberk S, Lima AR, Correia M, Batista R, Soares P, Máximo V, et al. Oncocytic thyroid neoplasms: from histology to molecular biology. Diagn Histopathol. 2019 May;25(5):154-65.

19 Kumar MS1. Pester RE, Chen CY, Lane K, Chin C, Lu J, Kirsch DG, Golub TR, Jacks T. Dicer1 functions as a haploinsufficient tumor suppressor. Genes Dev. 2009 Dec;23(23):2700-4.

20 Lambertz I, Nittner D, Mestdagh P, Denecker G, Vandesompele J, Dyer MA, et al. Monoallelic but not biallelic loss of Dicer1 promotes tumorigenesis in vivo. Cell Death Differ. 2010 Apr;17(4):633-41.

21 Wang W, Su X, He K, Wang Y, Wang H, Wang $\mathrm{H}$, et al. Comparison of the clinicopathologic features and prognosis of bilateral versus unilateral multifocal papillary thyroid cancer: an updated study with more than 2000 consecutive patients. Cancer. 2016 Jan; 122(2): 198-206.

22 Sancho JJ, Lennard TW, Paunovic I, Triponez F, Sitges-Serra A. Prophylactic central neck dissection in papillary thyroid cancer: a consensus report of the European Society of Endocrine Surgeons (ESES). Langenbecks Arch Surg. 2014 Feb;399(2):155-63. 